\title{
'IRDiRC Recognized Resources': a new mechanism to support scientists to conduct efficient, high-quality research for rare diseases
}

\author{
Hanns Lochmüller ${ }^{1}$, Yann Le Cam ${ }^{2}$, Anneliene H Jonker ${ }^{3}$, Lilian PL Lau ${ }^{3}$, Gareth Baynam ${ }^{4,5}$, Petra Kaufmann ${ }^{6}$, \\ Paul Lasko ${ }^{7}$, Hugh JS Dawkins ${ }^{8}$, Christopher P Austin ${ }^{6}$ and Kym M Boycott ${ }^{\star, 9}$ \\ on behalf of the IRDiRC Scientific Committees
}

\begin{abstract}
The International Rare Diseases Research Consortium (IRDiRC) has created a quality label, 'IRDiRC Recognized Resources', formerly known as 'IRDiRC Recommended'. It is a peer-reviewed quality indicator process established based on the IRDiRC Policies and Guidelines to designate resources (ie, standards, guidelines, tools, and platforms) designed to accelerate the pace of discoveries and translation into clinical applications for the rare disease (RD) research community. In its first year of implementation, 13 resources successfully applied for this designation, each focused on key areas essential to IRDiRC objectives and to the field of RD research more broadly. These included data sharing for discovery, knowledge organisation and ontologies, networking patient registries, and therapeutic development. 'IRDiRC Recognized Resources' is a mechanism aimed to provide community-approved contributions to RD research higher visibility, and encourage researchers to adopt recognised standards, guidelines, tools, and platforms that facilitate research advances guided by the principles of interoperability and sharing. European Journal of Human Genetics (2017) 25, 162-165; doi:10.1038/ejhg.2016.137; published online 26 October 2016
\end{abstract}

The International Rare Diseases Research Consortium (IRDiRC) was launched in 2011 to foster international research collaboration and investment in the field of rare disease (RD), with the objectives to contribute to the development of 200 new therapies and the means to diagnose most RDs by the year 2020. In 2013, IRDiRC issued its Policies and Guidelines document, composed of principles that its members agree to follow and recommendations made by IRDiRC Scientific Committees that offer advice on best practices for $\mathrm{RD}$ research. ${ }^{1}$ It emphasises the need for collaboration in $\mathrm{RD}$ research, the involvement of patients and their representatives in all relevant aspects of research, and the importance of sharing data and resources. On the basis of these principles and to contribute to its mission, IRDiRC introduced a quality indicator in March 2015 called 'IRDiRC Recommended' to highlight for researchers' resources which, if more broadly used, are expected to accelerate advances in $\mathrm{RD}$ research and development. $^{2}$

Earlier this year, this indicator was renamed 'IRDiRC Recognized Resources' 3 to convey more clearly the goal of the initiative: designating resources for $\mathrm{RD}$ research that have received recognition by researchers in the $\mathrm{RD}$ community. To obtain this label, resources undergo a peer-review process by IRDiRC Scientific Committee members and IRDiRC-independent researchers, who are often users of these resources themselves. The 'IRDiRC Recognized Resources' indicator is expected to promote the pace of discoveries and translation into clinical applications, in addition to encouraging the adoption of recognised standards, guidelines, tools, and platforms that facilitate research advances guided by the principles of interoperability and sharing. This paper presents an overview of the resources that have obtained the 'IRDiRC Recognized Resources' designation after the first year of its implementation.

\section{IRDIRC RECOGNIZED RESOURCES}

The application for 'IRDiRC Recognized Resources' is open to all project leaders of different resources (ie, standards, guidelines, tools, and platforms) designed to advance $\mathrm{RD}$ research and development. Applications are peer-reviewed with rolling submission based on a number of criteria (Table 1), and in particular, two mandatory requirements: resources must be within IRDiRC's focus and mission as defined in the IRDiRC Policies and Guidelines document, and have multinational connectivity and audience. Eligible resources include software, bioinformatics platforms, web services, RD data or biospecimen collections, international standards, and international guidelines (Table 2). Other resources, although acknowledged as important, are not eligible for the recognition: national, regional, or institutional biobanks and registries for RD; resources that are dedicated to a single disease entity; resources that could provide some utility for $\mathrm{RD}$, but are primarily designed for broader use; and commercial resources.

${ }^{1}$ Institute of Genetic Medicine, Newcastle University, Newcastle upon Tyne, UK; ${ }^{2}$ European Organisation for Rare Diseases (EURORDIS), Paris, France; ${ }^{3}$ IRDiRC Scientific Secretariat, Inserm US 14, Paris, France; ${ }^{4}$ Genetic Services of Western Australia, King Edward Memorial Hospital, Perth, Western Australia, Australia; ${ }^{5}$ Western Australian Register of Developmental Anomalies, Perth, Western Australia, Australia; ${ }^{6}$ National Center for Advancing Translational Sciences, National Institutes of Health, Bethesda, Maryland, USA; ${ }^{7}$ Department of Biology, McGill University, Montreal, Quebec, Canada; ${ }^{8}$ Department of Health, Office of Population Health Genomics, Public Health Division, Government of Western Australia, Perth, Western Australia, Australia; ${ }^{9}$ Department of Genetics, Children's Hospital of Eastern Ontario Research Institute, University of Ottawa, Ottawa, Ontario, Canada

*Correspondence: Dr KM Boycott, Department of Genetics, Children's Hospital of Eastern Ontario Research Institute, 401 Smyth Road, Ottawa, ON K1H 8 L1, Canada. Tel: +1 613 7377600 ext 4139; Fax: +1 613738 4822; E-mail: kboycott@cheo.on.ca

Received 31 August 2016; accepted 6 September 2016; published online 26 October 2016 
Table 1 Assessment criteria for 'IRDiRC Recognized Resources'

\begin{tabular}{|c|c|}
\hline Mandatory criteria & Additional criteria \\
\hline Within IRDiRC's focus and mission & Functional and accessible with minimal downtime \\
\hline \multirow[t]{8}{*}{ Multinational connectivity and audience } & Development and maintenance team \\
\hline & Clear and well-documented terms of use and license policies \\
\hline & Adheres to all relevant ethical and privacy policies and requirements \\
\hline & Process in place for quality control and life cycle management \\
\hline & Undergoes scientific peer review \\
\hline & Financially viable for the following 3 years \\
\hline & Documents its core impacts (eg, number of users, number of visits, etc) \\
\hline & Demonstrates relevant and ongoing activity in sharing and dissemination \\
\hline
\end{tabular}

Table 2 Eligibility for different types of 'IRDiRC Recognized Resources'

\begin{tabular}{ll}
\hline Eligible resources & Excluded resources \\
\hline Software, bioinformatics platforms, and web services & National, regional, or institutional biobanks RD, or a single disease entity \\
Data collections/biospecimen collections & National, regional, or institutional registries for RD, or a single disease entity \\
International standards & Resources with some utility for RD research, but primarily designed for broader use \\
International guidelines & Commercial resources \\
\hline
\end{tabular}

In its first year, 13 resources have been given the 'IRDiRC Recognized Resources' designation. These resources include three guidelines, five platforms, two reference databases, two standards, and an advisory committee (Table 3). These resources are hereby presented according to the area of focus in the context of the goals and objectives of IRDiRC.

\section{Facilitating international data sharing for discovery}

Four recognised resources have a strong emphasis on sharing approaches, aiming to advance knowledge about RD by encouraging global scientific collaboration while respecting ethical considerations. Two of these resources set out guidelines for legally- and ethicallygrounded sharing. The 'International Charter of principles for sharing bio-specimens and data' ${ }^{4}$ and the 'Framework for responsible sharing of genomic and health-related data ${ }^{5}$ aim to remove bottlenecks for effective sharing of $\mathrm{RD}$ data without compromising privacy, consent, and interest of individuals who participate and contribute their data for biomedical research. PhenomeCentral is a repository of RD patient phenotypic and genetic data, deposited by clinicians and scientists in this secure database to facilitate discovery; its collaborative model aims to understand the clinical spectrum and underlying mechanism of RD. ${ }^{6}$ In a similar manner, DECIPHER is a database that enables the depositing, analysing, and sharing of phenotype-linked plausible variation in patients with $\mathrm{RD}$, thereby empowering diagnosis and discovery. ${ }^{7}$ Both PhenomeCentral and DECIPHER are participants of the Matchmaker Exchange initiative, a collaboration between IRDiRC and the Global Alliance for Genomics and Health ${ }^{8}$ to enable genomic discovery through the exchange of phenotypic and genotypic profiles using a federated platform, ${ }^{9}$ thereby supporting the IRDiRC goal of providing a means to diagnose most RDs by 2020 .

\section{Knowledge organisation and ontologies}

Five widely used recognised resources are found in the area of knowledge organisation and ontologies. With respect to knowledge organisation, two databases facilitate access to $\mathrm{RD}$ information, Orphanet, and the Online Mendelian Inheritance in Man (OMIM).
Orphanet is a reference portal for $\mathrm{RD}$ and orphan drugs. ${ }^{10}$ It provides comprehensive information on research projects and their funders, registries and biobanks, platforms for research, clinical trials, a nomenclature and classifications of RDs, genes, and associated phenotypic and epidemiological data. OMIM is a widely used knowledge base of human genes and associated phenotypes comprised of over 23000 structured free-text entries. ${ }^{11,12}$ It captures published disease-gene relationships, and is a primary resource in human genetics for cataloguing and describing rare Mendelian diseases, and an essential tool for RD diagnosis.

Ontologies define a standard, controlled vocabulary for different fields in science and medicine to be utilised in data integration, organisation, search, and analysis. The Orphanet Rare Disease Ontology (ORDO), developed by Orphanet and the European Bioinformatics Institute, integrates different resources to provide a common framework for computational analysis of $\mathrm{RD}$; it presents a structured vocabulary for $\mathrm{RD}$, capturing relationships between diseases, genes, and other relevant features. ${ }^{13}$ The Human Phenotype Ontology (HPO) provides a standardised vocabulary to describe phenotypic abnormalities encountered in human disease, thus facilitating the exchange of clinical data and assisting $\mathrm{RD}$ diagnosis or searches for novel genes. ${ }^{14}$ The International Consortium of Human Phenotype Terminologies (ICHPT) has produced standard terms to enable interoperability between databases capturing clinical information, ${ }^{15}$ enabling connections between scientific resources for use in $\mathrm{RD}$ research.

\section{Networking patient registries}

Another necessary component to enable IRDiRC's mission is patient registries, which are organised databases of patient information, a critical tool for RD research. International collaboration regarding such databases is essential to assemble sufficient numbers of patients, for example to ascertain pathogenicity of rare genotypes, to carry out natural history studies, and identify participants for research and clinical trials. TREAT-NMD Patient Registries, a global network of national registries providing a single entry point for access to rare 
Table 3 IRDiRC Recognized Resources

\begin{tabular}{|c|c|c|}
\hline Resource name & Type & Description \\
\hline \multicolumn{3}{|l|}{ Facilitating international data sharing } \\
\hline $\begin{array}{l}\text { International Charter of principles for } \\
\text { sharing bio-specimens and data }\end{array}$ & Guideline & $\begin{array}{l}\text { The Charter provides recommendations for successful legally- and ethically-grounded sharing of } \\
\text { bio-specimens and data }\end{array}$ \\
\hline $\begin{array}{l}\text { Framework for responsible sharing of } \\
\text { genomic and health-related data }\end{array}$ & Guideline & $\begin{array}{l}\text { The Framework provides a principled and practical framework for the responsible sharing } \\
\text { of genomic and health-related data }\end{array}$ \\
\hline PhenomeCentral & Platform & $\begin{array}{l}\text { PhenomeCentral is a repository for secure sharing of phenotypic and genotypic data in the RD } \\
\text { community, thereby connecting patient profiles }\end{array}$ \\
\hline DECIPHER & Platform & $\begin{array}{l}\text { DECIPHER is a database and web-based platform enabling the deposition, analysis, and sharing of } \\
\text { phenotype-linked plausibly pathogenic variation in patients with RD }\end{array}$ \\
\hline \multicolumn{3}{|l|}{ Knowledge organisation and ontologies } \\
\hline Orphanet & Reference/database & Orphanet is a reference portal for information on RD and orphan drugs \\
\hline $\begin{array}{l}\text { Online Mendelian inheritance in man } \\
\text { (OMIM) }\end{array}$ & Reference/database & $\begin{array}{l}\text { OMIM is a database of human genes and genetic phenotypes comprised of over } 23000 \\
\text { structured free-text entries }\end{array}$ \\
\hline Orphanet rare disease ontology (ORDO) & Platform & $\begin{array}{l}\text { ORDO provides a structured vocabulary for RD, thereby aiming to define relationships between } \\
\text { diseases, genes, and other features of interest }\end{array}$ \\
\hline Human phenotype ontology (HPO) & Standard & HPO provides a standardised vocabulary of phenotypic abnormalities encountered in human disease \\
\hline $\begin{array}{l}\text { International Consortium of Human } \\
\text { Phenotype Terminologies (ICHPT) }\end{array}$ & Standard & $\begin{array}{l}\text { The ICHPT provides the community with a set of terms to describe phenotypic features to be } \\
\text { used by any terminologies to achieve interoperability between databases, in particular, to allow the } \\
\text { linking of phenotype and genotype databases for RDs }\end{array}$ \\
\hline \multicolumn{3}{|l|}{ Networking patient registries } \\
\hline TREAT-NMD patient registries & Platform & $\begin{array}{l}\text { The TREAT-NMD Patient Registries is a global network of national registries that provides } \\
\text { a unique entry point for access to rare neuromuscular disease patients worldwide }\end{array}$ \\
\hline \multicolumn{3}{|l|}{ Therapeutic development } \\
\hline $\begin{array}{l}\text { Standard operating procedures for } \\
\text { preclinical efficacy studies }\end{array}$ & Guideline & $\begin{array}{l}\text { Standard operating procedures for preclinical efficacy studies are a compilation of experimental } \\
\text { protocols to measure drug efficacy in models of neuromuscular disease }\end{array}$ \\
\hline Care and Trial Site Registry & Platform & $\begin{array}{l}\text { The Care and Trial Site Registry aims to assist pharmaceutical industry and clinical investigators } \\
\text { in deciding on clinical trial site location and in the identification of potential partners for future } \\
\text { research projects }\end{array}$ \\
\hline $\begin{array}{l}\text { TREAT-NMD Advisory Committee for } \\
\text { Therapeutics }\end{array}$ & Advisory Committee & $\begin{array}{l}\text { TREAT-NMD Advisory is a group of experts from various origins (academic, industry drug development, } \\
\text { patient representatives, and governmental representatives) that provide guidance on the translation } \\
\text { of therapeutic programs in rare neuromuscular diseases }\end{array}$ \\
\hline
\end{tabular}

neuromuscular disease patients worldwide, is such a recognised platform, ${ }^{16,17}$ and is expected to facilitate clinical trialling for this group of RDs.

\section{Therapeutic development}

IRDiRC aims to stimulate the development of 200 new therapies by 2020. TREAT-NMD has been active in confronting barriers in this space, producing resources to contribute to different phases of therapeutic development, from translation of basic research to clinical trials. Three additional resources from TREAT-NMD met IRDiRC's requirement regarding the adequate and timely exchange of scientific and regulatory information about clinical research, and are therefore recognised as important tools towards development of new therapies. First, its 'Standard Operating Procedures for preclinical efficacy studies' consist of a collection of experimental protocols for the most common outcome measures used in the assessment of drug efficacy in mammalian models of rare neuromuscular diseases. ${ }^{18,19}$ A wide use of such standardised protocols would contribute to improving robustness of preclinical results that serve to justify patient trials. One of the hurdles prior to initiating a clinical trial is the identification of trial sites capable of recruitment of sufficient number of patients, and qualified personnel to provide care and experience given a specific standard. The aim of the TREAT-NMD Care and Trial Site Registry is to help industry and clinical investigators identify and select trial sites and potential partners for clinical studies in neuromuscular and neurodegenerative diseases. ${ }^{20}$ The TREAT-NMD Advisory Committee for Therapeutics is composed of drug development experts from academia and industry, as well as representatives from patient and scientific research centres. This committee meets twice yearly to review and provide guidance on the translation and therapeutic development path for rare neuromuscular diseases with large unmet needs. ${ }^{21}$ Widespread adoption by the community of such recognised resources is expected to facilitate the IRDiRC goal to enable the development of 200 new therapies by 2020 .

\section{CONCLUSION}

After its first year, an assessment of the 'IRDiRC Recognized Resources' indicator shows that this initiative has highlighted 13 resources that focus on key IRDiRC priorities in advancing RD research: data sharing for discovery, knowledge organisation and ontologies, networking patient registries, and therapeutic development. It is expected that as more resources are highlighted and used by researchers, the pace of discovery and translation into the clinic will be further enhanced. Reciprocal recognition of resources between 
international efforts with overlapping goals (e.g. Human Variome Project) will be an important step forward. Moreover, as the research community converges on and adopts recognized standards, guidelines, tools, and platforms, the resulting interoperability will enable enhanced sharing, and thus accelerate advances in research and development across all RD.

\section{CONFLICT OF INTEREST}

The authors declare no conflict of interest.

\section{ACKNOWLEDGEMENTS}

The 'IRDiRC Recognized Resources' initiative has been supported by members of the IRDiRC Scientific Committees. Members include Fowzan Sami Alkuraya, Diego Ardigò, Michael Bamshad, Gert-Jan Boudewijn van Ommen, Anthony Brookes, Han Brunner, Angel Carracedo, Seng Cheng, Gema Chicano, Robin Conwit, Johan den Dunnen, Xavier Estivill, Jack Goldblatt, Stephen Groft, Shuling Guo, Adam Heathfield, Bartha Maria Knoppers, Jeffrey Krischer, Milan Macek, Sandrine Marreaud, Gert Matthijs, Woong-Yang Park, Samantha Parker, Asla Pitkänen, Catherine Rademaker, Pak-Chung Sham, Rumen Stefanov, Hendrik Stunnenberg, Domenica Taruscio, Josep Torrent i Farnell, Anne Zajicek, and Feng Zhang. We also acknowledge Ségolène Aymé, former Coordinator of the IRDiRC Scientific Secretariat, for her assistance in launching this initiative. The work was supported by the European FP7 contract, 'SUPPORT-IRDiRC' (No. 305207). CPA has contributed to this work in his capacity as Chair of the IRDiRC Consortium Assembly, not in his role as Director of the National Center for Advancing Translational Sciences.

1 IRDiRC. IRDiRC Policies \& Guidelines, 2013. Available at http://www.irdirc.org/reportsguidelines/policies-guidelines/ (accessed on 28 July 2016).

2 Aymé S: IRDiRC-recommended. Eur J Hum Genet 2016; 24: 955.

3 IRDiRC. IRDiRC Recognized Resources, 2016. Available at http://www.irdirc.org/ activities/irdirc-recognized-resources/ (accessed on 28 July 2016).

4 Mascalzoni D, Dove ES, Rubinstein $Y$ et al: International Charter of principles for sharing bio-specimens and data. Eur J Hum Genet 2015; 23: 721-728.

5 Global Alliance for Genomics and Health. Framework for responsible sharing of genomic and health-related data. Available at http://genomicsandhealth.org/framework (accessed on 28 July 2016).

6 Buske OJ, Girdea M, Dumitriu S et al: PhenomeCentral: a portal for phenotypic and genotypic matchmaking of patients with rare genetic diseases. Hum Mutat 2015; 36: 931-940.

7 Chatzimichali EA, Brent S, Hutton B et al: Facilitating collaboration in rare genetic disorders through effective matchmaking in DECIPHER. Hum Mutat 2015; 36: 941-949.
8 Global Alliance for Genomics and Health: GENOMICS. A federated ecosystem for sharing genomic, clinical data. Science 2016; 352: 1278-1280.

9 Philippakis AA, Azzariti DR, Beltran S et al: The Matchmaker Exchange: a platform for rare disease gene discovery. Hum Mutat 2015; 36: 915-921.

10 Rath A, Olry A, Dhombres F, Brandt MM, Urbero B, Ayme S: Representation of rare diseases in health information systems: the Orphanet approach to serve a wide range of end users. Hum Mutat 2012; 33: 803-808.

11 OMIM. Online Mendelian Inheritance in Man. Available at http://www.omim.org/ (accessed on 28 July 2016).

12 Amberger JS, Bocchini CA, Schiettecatte F, Scott AF, Hamosh A: OMIM.org: Online Mendelian Inheritance in Man (OMIM), an online catalog of human genes and genetic disorders. Nucleic Acids Res 2015; 43: 789-798.

13 Vasant D, Chanas L, Malone J et al: ORDO: an ontology connecting rare disease. Epidemiology and genetic data, 2014. Available at: http://www.orphadata.org/cgi-bin/ inc/ordo_orphanet.inc.php (accessed on 28 July 2016).

14 Köhler S, Doelken SC, Mungall CJ et al: The Human Phenotype Ontology project: linking molecular biology and disease through phenotype data. Nucleic Acids Res 2014; 42: 966-974.

15 ICHPT. Proposal of a core set of terms to describe human phenotypes by the International Consortium of Human Phenotype Terminologies, 2015. Available at http://www.irdirc.org/wp-content/uploads/2015/09/Proposal-International-Consortium-of-Human-Phenotype-Terminologies.pdf (accessed on 28 July 2016).

16 Sárközy A, Bushby K, Béroud C, Lochmüller H: 157th ENMC International Workshop: patient registries for rare, inherited muscular disorders 25-27 January 2008 Naarden, The Netherlands. Neuromuscul Disord 2008; 18: 997-1001.

17 Bladen CL, Rafferty K, Straub V et al: The TREAT-NMD Duchenne muscular dystrophy registries: conception, design, and utilization by industry and academia. Hum Mutat 2013; 34: 1449-1457.

18 TREAT-NMD. Standards in preclinical efficacy studies. Available at http://www. treat-nmd.eu/research/preclinical/preclinical-efficacy-standards/ (accessed on 28 July 2016).

19 Willmann R, De Luca A, Benatar M et al: Enhancing translation: guidelines for standard pre-clinical experiments in mdx mice. Neuromuscul Disord 2012; 22: 43-49.

20 Rodger S, Lochmüller H, Tassoni A et al: The TREAT-NMD care and trial site registry: an online registry to facilitate clinical research for neuromuscular diseases. Orphanet $J$ Rare Dis 2013; 8: 171-183.

21 Heslop E, Csimma C, Straub V et al: The TREAT-NMD advisory committee for therapeutics (TACT): an innovative de-risking model to foster orphan drug development. Orphanet J Rare Dis 2015; 10: 49-55.

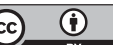

This work is licensed under a Creative Commons Attribution 4.0 International License. The images or other third party material in this article are included in the article's Creative Commons license, unless indicated otherwise in the credit line; if the material is not included under the Creative Commons license, users will need to obtain permission from the license holder to reproduce the material. To view a copy of this license, visit http:// creativecommons.org/licenses/by/4.0/

(C) The Author(s) 2017 